Archives

16 | 1996

Pour une histoire comparée du vœu

\title{
Vœu chevaleresque et vœu de croisade dans le roman de Tirant lo Blanc (1460-1490)
}

La fin de l'empire chrétien d'Orient

Dominique de Courcelles

\section{CpenEdition}

Journals

Édition électronique

URL : http://journals.openedition.org/ccrh/2652

DOI : $10.4000 /$ ccrh.2652

ISSN : 1760-7906

Éditeur

Centre de recherches historiques - EHESS

Édition imprimée

Date de publication : 16 avril 1996

ISSN : 0990-9141

Référence électronique

Dominique de Courcelles, « Vœu chevaleresque et vœu de croisade dans le roman de Tirant lo Blanc (1460-1490) », Les Cahiers du Centre de Recherches Historiques [En ligne], 16 | 1996, mis en ligne le 27 février 2009, consulté le 20 avril 2019. URL : http://journals.openedition.org/ccrh/2652 ; DOI : $10.4000 /$ ccrh. 2652

Ce document a été généré automatiquement le 20 avril 2019

Article L.111-1 du Code de la propriété intellectuelle. 


\title{
Vœu chevaleresque et vœu de croisade dans le roman de Tirant lo Blanc (1460-1490)
}

\author{
La fin de l'empire chrétien d'Orient
}

Dominique de Courcelles

1 Dans la littérature catalane de la fin du Moyen Âge, le roman de chevalerie intitulé Tirant lo Blanc est une des œuvres les plus célèbres. Miguel de Cervantés, qui le lit en castillan au $\mathrm{XVI}^{\mathrm{e}}$ siècle, estime qu'il est "parmi la foule innombrable et vaine des livres de chevalerie » « de par son style le meilleur roman du monde $»^{1}$. Il est traditionnellement attribué à un chevalier valencien, querelleur et batailleur, qui ne résida presque jamais à Valence et parcourut l'Europe, Joanot Martorell, né vers 1412-1413 à Gandie et mort à Valence en 1468. Les lettres de bataille de Joanot Martorell ${ }^{2}$ établissent qu'il a séjourné à la cour d'Angleterre dans les années 1438-1439, dans le royaume de Portugal, dans le royaume de Naples et en Sicile.

2 Écrit sans doute entre 1460 et 1468 , ce roman raconte les aventures d'un chevalier breton, dénommé Tirant lo Blanc, errant loin de son pays en quête du véritable « honneur » de chevalerie et amené, en raison de son éclatante bravoure, à se rendre à Constantinople afin de combattre sans répit les Maures qui cherchent à s'emparer de l'empire chrétien d'Orient. A l'époque où le chevalier valencien Joanot Martorell parcourt l'Europe et compose son roman, les relations entre le monde occidental chrétien et le monde oriental musulman, qui menace Vienne par les Balkans et la vallée du Danube, deviennent un fait majeur de la civilisation. L'Empire ottoman s'intègre à l'horizon européen. Ces relations sont ambivalentes, faites à la fois de crainte et d'agressivité, d'admiration, de curiosité et de contacts pacifiques. C'est la puissance ducale de Bourgogne avec le prestigieux Ordre chevaleresque de la Toison d'Or qui paraît tenir un rôle déterminant dans la défense et la reconquête de l'empire chrétien d'Orient.

3 Joanot Martorell, comme tout chevalier qui a séjourné dans les différentes cours européennes, ne peut pas ignorer que c'est surtout au duc de Bourgogne, le « grand duc d'Occident", et à ses chevaliers que s'adressent les empereurs de Constantinople, 
menacés par les turcs. L'auteur anonyme du Livre des faits de Jacques de Lalaing, ouvrage bien diffusé en Espagne, rapporte qu'un ambassadeur byzantin, venu trouver le duc Philippe le Bon à Châlon-sur-Saône, s'exprime en ces termes : « Et disait que, si par le duc de Bourgogne n'était secouru et aidé, il ne voyait nul prince chrétien qui eut volonté de bailler secours pour aider à défendre la chrétienté, laquelle un chacun jour le Turc s'efforçait de tout son pouvoir de vouloir détruire et anéantir »3. Le grand duc d'Occident n'est-il pas roi sans en avoir le titre ? Philippe le Bon déclare fièrement en $1464:$ «Je veux bien que chacun sache que, si j'eusse voulu, je fusse roi." Après la prise de Constantinople, le 29 mai 1453, l'empire chrétien d'Orient s'effondre. Alors que tout l'Occident pleure et s'interroge sur cette disparition, le Vœu du Faisan formulé à Lille par le duc de Bourgogne et les nobles de son entourage au cours de fêtes somptueuses proclame l'intention de la reconquête, glorifie la puissance bourguignonne, frappe durablement les imaginaires contemporains.

Telles sont les conditions dans lesquelles la politique comme la littérature s'emparent du vœu chevaleresque, du vœu de croisade. Je rappellerai d'abord les enjeux du Vœu du Faisan, puis je comparerai les vœux chevaleresques des nobles de l'entourage du duc de Bourgogne avec ceux de Tirant et de ses compagnons dans le roman de Tirant lo Blanc.

\section{Le vœu du Faisan}

5 Tout part de cet événement mis en scène par le duc de Bourgogne et ses conseillers : geste singulier, destiné à interrompre le déroulement de l'histoire, l'histoire de l'empire chrétien d'Orient et celle du duc de Bourgogne.

6 "Dimanche passé mondit seigneur le duc fist une feste si haulte, si solempnelle et si pompeuse que je cuide que de aige d'homme vivant n'a poinct esté faite la pareille $»^{4}$. $C^{\prime}$ 'est ainsi que Jean de Molesme, secrétaire du duc Philippe le Bon, parle du banquet du Vœu du Faisan dans une lettre aux maires et échevins de Dijon. Et il ajoute: «Ce n'a poinct esté seulement que un grand banquet duquel sera mémoire cy-après tant en chrestienté qu'ailleurs.» Les chroniqueurs du duc expliquent que "beaucoup de chevaliers et de dames de grande maison » étaient venus de loin, «les uns par mer, les autres par terre, pour voir ceste feste, dont il s'estoit publié de grandes nouvelles $»^{5}$.

7 Si l'empire chrétien d'Orient s'est révélé périssable, le duc de Bourgogne Philippe le Bon est bien décidé à doter de permanence son action, ses paroles, sa maison, en les faisant objet de mémoire, objet de récit. La grandeur humaine de la maison ducale et du duc, vue dans l'activité futile et peu durable d'un somptueux banquet, acquiert par la parole, celle $\mathrm{du}$ duc et des nobles qui l'entourent, un renom immortel. Cette parole, c'est le Vœu du Faisan.

8 Né en 1396, l'année où son père Jean sans Peur subit dans l'orientale Nicopolis une cruelle défaite, Philippe le Bon appartient à la famille royale de France. Sa puissance en Bourgogne et en Flandre, accrue par la force, l'argent ou la diplomatie, lui vaut la méfiance justifiée du roi Charles VII. L'épopée de Jeanne d'Arc a lieu en 1429-1430, les armées françaises ne reprennent Rouen et Bordeaux aux Anglais qu'en 1452 et 1453, aucune paix n'est signée entre la France et l'Angleterre, et le danger d'une reprise de la guerre ou d'un accord entre les Anglais et les Bourguignons persiste. En 1453, Philippe le Bon achève de réduire les deux plus grandes villes de Flandre par les armes: Bruges en 1438 et surtout Gand en 1453. Il tend désormais à unifier son système de gouvernement. 
La puissance diplomatique et militaire du duc de Bourgogne en Europe peut paraître l'emporter sur celle du roi Charles VII. S'il entend bien mener des actions en Orient, ce n'est pas pour délivrer Jérusalem, car le désastre de Nicopolis a montré que les risques étaient trop grands, mais pour préserver ses intérêts aux marges orientales du monde chrétien où aboutissent les routes caravanières traditionnelles du commerce avec l'Asie ${ }^{6}$.

C'est à l'occasion de son mariage avec Isabelle de Portugal, fille de Jean ${ }^{\text {er }}$ de Portugal et de l'Anglaise Philippa de Lancaster, en 1429, que Philippe le Bon a institué l'Ordre de la Toison d'Or. Il est désormais allié à la maison de Portugal - Alphonse V, neveu d'Isabelle, règne de 1438 à 1481 - et à la maison d'Aragon et de Naples - en particulier à Alphonse le Magnanime, roi d'Aragon et de Naples; Isabelle de Portugal n'oublie pas ses liens avec l'Angleterre et souhaite le mariage anglais de son fils Charles. Le duc est surtout sensible aux événements de l'est, à tout ce qui se passe au contact des Slaves et des musulmans ; la duchesse est sensible à ce qui se passe à l'ouest, en particulier au Maroc, domaine de l'expansion portugaise. La cour de Bourgogne est alors à la fois bourguignonne et portugaise, puisque la duchesse est accompagnée d'un nombre important d'hommes et de femmes de son pays d'origine. L'Ordre de la Toison d'Or acquiert rapidement un immense prestige.

dre est une fraternité chrétienne vouée à la défense de la foi, dans une fidélité absolue à l'égard du chef de l'ordre, le duc de Bourgogne. Ses statuts ont été proclamés à Lille le 30 novembre 1431, jour de la fête de saint André apôtre, patron des ducs. L'ordre ne comporte à l'origine que vingt-quatre chevaliers (le nombre est élevé à trente en 1433), de la plus haute noblesse, ce qui lui donne un grand prestige.

11 Arrivant dans sa fidèle ville de Lille en janvier 1454, Philippe le Bon souhaite évidemment fêter sa victoire sur Gand. Beaucoup de nobles sont rassemblés à Lille pour le mariage princier d'Isabelle d'Étampes, fille du comte d'Étampes, avec le duc de Clèves. Le comte est le cousin fidèle de Philippe le Bon et le duc de Clèves est son neveu.

12 "Grans chieres et grans festiemens » ont donc lieu à Lille. Les chevaliers se donnent véritablement à voir et admirer, cependant que la victorieuse grandeur du duc est manifestée. C'est alors que, dans le déroulement de la fête, font irruption par représentation les deux grandes puissances symboliques du monde occidental, le pape et l'empereur. Leurs ambassadeurs sollicitent le duc de Bourgogne - et non le roi de France - par deux récits successifs qui concourent à signifier la majesté et la puissance du duc. Le récit de l'ambassadeur pontifical est dramatique: il évoque la profanation de l'église Sainte-Sophie, particulièrement chère aux croisés, il raconte les viols et les meurtres perpétrés par les infidèles sur les chrétiens; le conquérant de Constantinople, selon le récit, est le fils du sultan qui a fait prisonnier le duc Jean de Bourgogne, c'est-àdire le père de Philippe le Bon, à Nicopolis en 1396 : il y a erreur généalogique, mais le rappel de Nicopolis doit engager Philippe le Bon, vainqueur des Gantois et désormais libre, à venger son père. Le récit de l'ambassadeur de l'empereur évoque le rassemblement des princes allemands à Ratisbonne en vue de la lutte commune contre les Ottomans et de la reconquête; le duc est prié de venir diriger l'entreprise. Du roi de France, suzerain du duc de Bourgogne et son parent, il n'est fait mention ni par l'ambassadeur du pape ni par celui de l'empereur.

13 « Pour esmouvoir les seigneurs et nobles hommes de ses pays et ses subjectz à servir Dieu en ceste partie et que de leur dévocion, sans contrainte, ils entrassent au sainct voyage si prit conseil de publier son emprise par voye de grande assemblée » (Olivier de La Marche). Cette grande assemblée est le banquet du Vœu du Faisan. Les fidèles du duc 
organisent le déroulement de la cérémonie : Jean de Lannoy, chevalier de la Toison d'Or, le chroniqueur officiel Olivier de La Marche, le chancelier Nicolas Rolin, le premier chambellan Antoine de Croy. Pouvoir et mémoire concourent à l'élaboration de la cérémonie du Vœu.

Dans un décor luxueux garni d'animaux rares, d'automates et de fontaines, animé de musiciens et de chanteurs, dominé par la statue de saint André, la proche famille ducale et les personnages importants des États de Bourgogne prennent place autour de trois tables. Outre les convives, de nombreux spectateurs sont présents. L'abondance des mets et des spectacles ou entremets, intermèdes mimés, parlés ou dansés, est tout à fait remarquable. L'un des derniers intermèdes, l'histoire de Jason, le héros de la Toison d'Or, est présentée en trois tableaux. La Toison d'Or est ce prix merveilleux que remportent Jason et ses compagnons, les Argonautes, grâce à leur conduite noble et courageuse. La référence à Jason est évidemment à mettre en relation avec l'Ordre de la Toison d'Or créé par le duc.

Enfin entre dans la salle du banquet un géant "vêtu comme un sarrazin de Grenade " conduisant un éléphant (une machine) sur lequel se trouve une forteresse. Dans cette forteresse une femme en deuil, personnage joué par le chroniqueur Olivier de La Marche, a une robe de satin blanc, un manteau noir et une coiffe semblable à celle des béguines. Elle se nomme : elle est l'Église qui demande l'aide du duc, « doyen des pairs de France ", et des chevaliers, "vous qui portez la Toison ». Le roi d'armes ou héraut Toison d'Or portant un faisan s'avance alors, accompagné de deux demoiselles et deux chevaliers. Il rappelle au duc la coutume qui veut que soit présenté un oiseau, paon ou faisan, sur lequel sont prononcés des vœux. Le duc dit seulement : « Je voue à Dieu mon créateur, à la glorieuse Vierge Marie, aux dames et au faisan, que je feray et entretenray ce que je baille par escript ». Puis il donne au héraut un billet que celui-ci lit à haute voix ; c'est le vœu entier de croisade de Philippe le Bon.

" Je veue à Dieu mon créateur, tout premièrement, et à la très glorieuse Vierge, sa mère, et en apprez aux dames et au faisant, que se le plaisir du très crestien et très victorieux prince, monseigneur le Roy, est de entreprenre et expozer son corps pour la deffence de la Foy crestienne et résister à la dampnable emprinse du Grand Turcq des infidèles, et lors se je n'ay lealle ensoinne de mon corps, je le serviray en ma personne de ma puissance, audit saint voyage, le mieulx que Dieu me donra sa grâce... Et se durant ledit saint voyage je puis, par quelque voye ou manière que ce soit, savoir ou congnoistre que ledit Grand Turc ait volenté d'avoir à moy afaire corps à corps, je, pour ladicte Foy crestienne soubstenir, le combatteray, à l'ayde de Dieu tout puissant et de sa douche mère, lesquelz je appelle tousjours en mon aide. Fait à Lille, le XVII jour de février, l'an de grâce Nostre Seigneur mil CCCC cinquante et trois » (Chronique de Mathieu d'Escouchy).

Le duc ne parle pas lui-même, il fait lire par le héraut Toison d'Or son vœu écrit, comme s'il s'agissait d'une lettre de bataille, d'une provocation, d'un défi avant la lutte. Le vœu commence comme un vœu de croisade, prenant Dieu et la Vierge à témoins, et se poursuit comme un vœu courtois, prenant les dames et le faisan à témoin.

Le duc proteste de son obéissance au roi de France, a soin de soumettre sa décision de croisade à celle du roi, tout en soulignant qu'il est prêt à le remplacer, s'il le faut, à la tête de la croisade ; ces précautions oratoires sont renouvelées à trois reprises. La conclusion $\mathrm{du}$ vœu du duc est très révélatrice : en envisageant que le Grand Turc souhaite se battre corps à corps avec lui, il se pose en égal physique et politique du chef des Turcs. Il n'est pas question du roi de France. 
L'Église remercie le duc de Bourgogne en ces termes :

«Dieu soit servy et loé hautement,/ De toy, mon fils, doyen des pers de France,/ Ton très hault vœu m'est tel enricement/ Qu'il me semble que je suis clèrement/ De tous mes maux à plaine délivrance;/ Par tout m'en voy requérir aliance/ Et prie à Dieu qu'il t'ottroie la grâce/ Que ton désir à son plaisir se face ».

Le duc est proclamé fils de l'Église, plus pair que tous les pairs de France ; sa parole a le pouvoir de donner à l'Église un clair semblant de salut; son désir et le plaisir de Dieu se rejoignent. Le vœu de croisade a déjà trouvé son efficacité. L'Église s'adresse alors aux chevaliers qui entourent le duc afin de les encourager à suivre l'exemple ducal :

«O vous, princes, chevalliers, nobles hommes,/ Voyez patron pour haulx fais entreprendre ;/ Froissiez vos aises, atoumissiez vos souaiz,/ Levez vos mains tandis que nous y sommes,/ Offrez à Dieu ce que lui devez rendre./ Sy prens congé, je ne me puis deffendre,/ Mais vois tirant la terre crestienne,/ Pour Dieu servir et abréger ma peine ».

19 Les princes, chevaliers, nobles hommes, c'est-à-dire toute la noblesse haute ou basse, sont invités à vouer à leur tour en levant la main. L'Église elle-même conseille au duc qui a voué de faire prêter serment aux nobles présent au banquet. Mais il est vrai que l'Église a les traits d'Olivier de La Marche, le chroniqueur aimé du duc, chargé de glorifier la dynastie bourguignonne. Le géant repart avec l'éléphant et la forteresse dans laquelle demeure l'Église toujours captive. Dans l'émotion et l'enthousiasme, certains nobles s'engagent immédiatement. Ils sont si nombreux que le duc fait crier à Toison d'Or que tous ceux qui veulent faire un vœu de croisade doivent l'écrire et le donner le lendemain. «Il les tenroit [les vœux] pour vaillables et agréables, comme se ilz eussent esté fais en sa présence.»

20 Le récit des chroniqueurs s'interrompt pour faire place à l'énoncé des vœux écrits, temps manqué par l'énoncé du cérémonial lui-même du banquet du Faisan :

« Mais ce nonobstant se veul-je baillier la déclaracion des veulx qui furent fais à ceste heure, à cause de la lamentacion et pitié de l'Église, et me soit pardonné, se je ne les mès sy bien par ordre qu'il appartenroit; et se faulte $\mathrm{y} a, c^{\prime}$ est par ignorance et peu savoir » (Chronique de Mathieu d'Escouchy).

21 "Valables et agréables", les vœux remis au duc de Bourgogne ne sont donc pas proclamés, ils sont secrets, tout en prenant à témoin de leur écriture Dieu, la Vierge, les dames et le faisan, parfois seulement les dames et le faisan. Ils sont très précisément et fidèlement inscrits par les chroniqueurs dans la mémoire historique de la Maison de Bourgogne. Ces vœux marquent, par le secret de vassal à suzerain, le renouvellement du lien de fidélité, du lien d'alliance. La fidélité de l'écriture renvoie à la fidélité des chevaliers qui ont signé les vœux. Il est essentiel que le secret des vœux écrits soit publié en tant que secret, engagement d'homme à homme, dans les chroniques destinées à glorifier la puissance des ducs de Bourgogne.

Il est remarquable que, dans les mois qui suivent le banquet du 17 février 1454, le duc de Bourgogne fait organiser par des membres de sa famille ou par ses proches conseillers des assemblées à Arras, Mons, Bruges, afin de prolonger les engagements pris et demander des vœux à tous les nobles d'Artois, Hainaut et Flandre. Rien n'indique qu'il en ait demandé en Bourgogne, le cœur de la puissance ducale, et les chevaliers de la Toison d'Or sont très peu nombreux à vouer; de fait, le duc ne saurait mettre en cause leur absolue fidélité. Comment les autres nobles pourraient-ils refuser de prononcer le vœu que leur demande leur suzerain? Un chevalier ne peut refuser de combattre pour la foi chrétienne et la chrétienté. L'invocation mêle les références religieuses : Je voue à Dieu, à la Vierge 
Marie, et les références courtoises : Je voue aux dames, au faisan. Mais le vœu lui-même est d'abord déclaration d'obéissance au suzerain, obéissance du duc au roi de France, obéissance des nobles au duc. L'engagement des nobles est plus la manifestation d'une soumission à la volonté et au pouvoir du duc de Bourgogne qu'un vœu de croisade proprement dit; ainsi le duc de Clèves voue aux dames et au faisan que «je serviray monseigneur mon oncle s'il lui plaist » et le seigneur de Ligne «voue aux dames et au faisan en brief langage qu'il volra estre obéissant à son très redouté seigneur et souverain prince, comme son vray et loyal sujet ». Seul le comte de Saint-Pol ne parle que de son obéissance au roi de France, se posant en égal du duc de Bourgogne, mais il est bientôt sévèrement humilié, car, lorsqu'il organise à Cambrai une fête de la Licorne, en réponse aux fêtes de Lille, le duc et les siens n'y paraissent pas et la fête de la Licorne est un échec. Philippe le Bon obtient donc, après la victoire sur les villes rebelles, une nouvelle victoire qui vient compléter de façon indispensable la précédente : le renforcement de ses liens avec les nobles de ses États. La narration peut reprendre :

«Apprès les veux fais, comme j'ay dit, le banquet qui longuement avoit duré, fut assouvy... » (Chronique de Mathieu d'Escouchy). occidentale, n'a en fin de compte guère déployé son effective puissance afin de reconquérir l'empire chrétien d'Orient. La croisade du duc n'est jamais partie. Les Occidentaux ont-ils définitivement renoncé à l'Orient? Admettent-ils désormais que le monde ottoman, musulman, fait partie de l'espace européen ? Bientôt François $\mathrm{I}^{\mathrm{er}}$ traitera avec la puissance ottomane contre Charles Quint. 


\section{Les vœux de Tirant et ses compagnons dans le roman de Tirant lo Blanc à la fin du $x v^{\mathrm{e}}$ siècle}

Le roman de Tirant s'ouvre par la mention de l'adresse au «Prince don Ferrando de Portugal ». Martorell indique ensuite que le livre est la traduction en langue vulgaire valencienne d'une version portugaise qui est elle-même la traduction d'un texte anglais original. Ce texte en langue valencienne réalisé à partir de la version portugaise d'un original anglais ne ferait-il pas en quelque sorte écho à la double origine anglaise et portugaise de la duchesse de Bourgogne Isabelle de Portugal, ne désignerait-il pas, par résonance, par glissement, les objectifs orientaux du duc de Bourgogne et de l'Ordre de la Toison d'Or institué à l'occasion du mariage portugais?

L'infant Ferran de Portugal, né en 1433, frère du roi Alphonse V et neveu aimé de la duchesse de Bourgogne, est "roi en attente d'être roi » du Portugal, pour reprendre l'expression de Martorell, entre 1438, date de la mort de son père, et 1451, date de la naissance d'un héritier de son frère. Il ne l'est donc plus en 1460, date à laquelle Martorell affirme avoir commencé son roman. A partir de 1450, Ferran qui est en conflit avec son frère quitte la cour de Portugal et se rend en Afrique du Nord et à Naples, désireux de combattre les Maures et d'acquérir la gloire.

Aucun manuscrit du roman de Tirant lo Blanc n'est conservé. La première édition de 1490 est close par un colophon qui apprend au lecteur que l'écrivain mentionné dans le titre et la dédicace du livre, « le magnifique et vertueux chevalier Mossen Joanot Martorell », « en raison de sa mort, n'a pu achever de traduire que trois parties du livre» et que «la quatrième partie, qui est la fin du livre, a été traduite, sur les prières de la noble dame Isabelle de Lloris, par le magnifique chevalier Mossen Martí Joan de Galba ». Le colophon prend fin sur la mention de l'achèvement de l'impression du livre : le 20 novembre 1490 à Valence. Une omission est remarquable : celle du nom de l'imprimeur Nicolau Spindeler.

Aussi apparaît-il bien qu'en affirmant la double origine anglaise et portugaise de son roman en langue valencienne, en introduisant un second auteur-traducteur, en nommant Isabelle de Lloris - qui ne serait en tant que telle peut-être qu'un leurre (loris, en ancien français, loris, en ancien catalan, signifie leurre) renvoyant à une seconde Isabelle qui est peut-être la duchesse de Bourgogne -, en choisissant pour destinataire un personnage en mal d'identité royale, un prince portugais errant du Portugal à l'Afrique, à Naples et à Barcelone, l'écrivain donne un indice d'une sorte de modèle de l'action qui se déploie dans le roman par redoublement : de l'Occident à l'Orient, de l'Angleterre et du Portugal à la Méditerranée à la fois occidentale et orientale, soit le domaine où le puissant duc de Bourgogne Philippe le Bon, appuyé par les chevaliers de l'Ordre de la Toison d'Or, cherche lui-même à déployer son influence et son prestige, à mesurer les forces et les méthodes de l'ennemi, à éviter de nouveaux désastres.

Tirant lo Blanc est écrit postérieurement au siège de Rhodes du 10 août au 18 septembre 1444, dont Martorell peut avoir d'amples informations grâce à son ami le corsaire valencien Jaume de Vilaragut, rentré d'Orient en 1447 et particulièrement lié au célèbre chevalier bourguignon, Geoffroy de Thoisy ${ }^{7}$. Rhodes est une île où se trouvent en grand nombre les Catalans et les Valenciens, tant comme chevaliers de l'Ordre de SaintJean que comme marchands, banquiers, marins, etc. A Rhodes se trouvent également de très nombreux Bourguignons qui sont les alliés naturels des Catalans et des Aragonnais 
contre les Français et les Sarrasins. Les Gênois, ennemis du roi d'Aragon Alphonse le Magnanime, accusent les chevaliers de l'ordre d'être favorables au roi et ils aident le sultan d'Égypte dans le siège de Rhodes. Le duc de Bourgogne est particulièrement attentif au sort de Rhodes. Il est notable que les galères qu'il arme en Méditerranée sont avant tout destinées à aider les chevaliers de Rhodes contre les Égyptiens. En 1465, le duc, qui n'a pu partir lui-même en croisade, donne aux Hospitaliers de Rhodes une énorme somme d'argent qui leur permet de construire leur bastion de Rhodes et d'y demeurer jusqu'en 1522.

31 Les aventures orientales du héros Tirant commencent précisément lorsqu'il décide, à la suite de l'appel du Grand Maître des chevaliers de Rhodes, de quitter sa terre d'origine, l'arthurienne Bretagne, et de se rendre à Rhodes afin de secourir les chevaliers assiégés. Il passe d'abord par le Portugal avant de traverser le détroit de Gibraltar et d'affronter pour la première fois les Maures. Le Portugal est donc la première étape du voyage de Tirant vers l'Orient. Angleterre, Bretagne et Portugal, ces terres les plus occidentales de l'Occident, constituent ainsi, au point de départ des aventures de Tirant, leur véritable condition de possibilité.

Joanot Martorell attribue à son héros le rôle qui est reconnu au " grand duc d'Occident ", sans jamais mentionner la Bourgogne, ni son duc, ni l'Ordre de la Toison d'Or. Dans le Tirant, l'empereur de Constantinople écrit au roi de Sicile afin de lui demander de lui envoyer en secours Tirant, chevalier de l'Ordre de la Jarretière, qui a été déclaré par le roi d'Angleterre le «meilleur chevalier du monde » et qui lui paraît le seul homme capable de repousser les turcs (chap.115-116). Tirant, arrivant à Constantinople, devient «grand capitaine » de l'empire byzantin.

Si l'ordre bourguignon de la Toison d'Or n'est jamais évoqué dans le roman, ses principales caractéristiques sont attribuées à l'Ordre de la Jarretière. Les chapitres ou statuts de l'Ordre de la Jarretière sont au nombre de cinq. Les quatre premiers chapitres, à l'imitation de la Table Ronde, donnent des règles de conduite : seul un chevalier peut appartenir à la fraternité de l'ordre; il ne doit jamais se séparer de son roi et seigneur naturel ; il doit aider et protéger les veuves, les femmes mariées et les jeunes filles ; il ne doit jamais fuir devant l'ennemi. «Le cinquième chapitre est le suivant, explique le roi d'Angleterre à Tirant: si le roi d'Angleterre entreprend d'aller conquérir la terre sainte de Jérusalem, en quelque état que se trouve le chevalier, qu'il soit blessé ou malade, il est tenu de rejoindre par mer ma fraternité, car la conquête de Jérusalem m'appartient à moi qui suis roi d'Angleterre et à nul autre.» Comme dans les statuts de l'Ordre de la Toison d'Or, la fidélité au chef de l'ordre se conjugue avec les conduites chevaleresques et la croisade. Le roi Henri d'Angleterre est-il ici, par glissement, une figure du grand duc d'Occident?

Sauver Constantinople du péril turc est un topos qui existe dans la littérature bien avant la chute de l'empire byzantin. La cour de Bourgogne, point de départ des chevaliers bourguignons vers Constantinople ou les îles franques de Méditerranée, est aussi le lieu d'élaboration des chroniques et des mémoires chevaleresques, dans la perspective de la sauvegarde ou de la reconquête de l'Orient. Ne rejoint-elle pas, par son rôle langagier, le roman de Tirant lo Blanc?

C'est ainsi que, dans ses Anciennes Chroniques d'Angleterre, Jean de Wavrin, chroniqueur des ducs de Bourgogne et brillant chevalier qui a apporté son aide à l'empereur byzantin et séjourné à Constantinople ${ }^{8}$, reprend, exactement comme l'auteur de Tirant, le roman 
anglo-normand de Guy de Warwyck qui date du XIII ${ }^{\mathrm{e}}$ siècle : l'empereur de Constantinople, menacé par le sultan de Babylonie, est sauvé du péril par Guy de Warwyck et lui accorde sa fille comme épouse, ce que le héros refuse par fidélité à sa dame. Après avoir rappelé cette histoire, Jean de Wavrin évoque longuement la personnalité de « Messire Jehan de Hongnac dit le Blanc chevalier ", qui constitue dans la chronique à la fois une figure et un modèle, parmi d'autres illustres, du puissant duc de Bourgogne et des chevaliers de la Toison d'Or. En 1448, Jean Hunyade remporte sur les Turcs une grande victoire et éloigne pour quelque temps le péril turc de Constantinople et des îles grecques : « Ainsi demeura aux chrétiens la terre, le peuple et grande partie du pays de l'occident de Grèce ${ }^{9}$. Mais il meurt, mortellement blessé, en 1456, après une nouvelle victoire à Belgrade. Jean Hunyade, père du futur roi de Hongrie Mathias Corvin, est valache; ses contemporains le dénomment Valachus ou Balachus, mot qui se maintient en Occident sous la forme Blac ou Blach, ce qui devient Blanc ou Blanch, Bianco ${ }^{10}$. Jean Hunyade est l'un des principaux modèles de Tirant lo Blanc. Par la figure dramatique et célèbre de Jean Hunyade, le grand duc d'Occident rejoint le héros du roman valencien.

Le Vœu du Faisan est doublement présent dans le roman de Tirant lo Blanc. Le héros formule à deux reprises un vœu et, à deux reprises, il est imité par ses compagnons qui sont ses parents et ses alliés. Le premier vœu de croisade de Tirant est proclamé après les noces de l'infante de Sicile et du fils du roi de France, en présence du roi de France, dont les nefs et les galères se trouvent devant Tripoli de Syrie; en un intermède Tirant a dû traverser la mer avec ses compagnons pour se rendre auprès du roi. Le second vœu de Tirant est proclamé lors des fêtes données par l'empereur en son palais de Constantinople ${ }^{11}$; l'intermède qui précède directement le vœu est constitué par l'arrivée dans le port de Constantinople du roi Arthur et de la fée Morgane sur une nef merveilleuse «sans mât ni voile », tout à fait semblable à l'un des automates de la fête du Vœu du Faisan ${ }^{12}$.

Selon la chronique de Mathieu d'Escouchy, Geoffroy de Thoisy, le chevalier navigateur bien connu des Catalans, prononce le vœu suivant: "Je fais vœu que je serai à mon pouvoir des premiers prêts pour aller au saint voyage avec Monseigneur et des derniers qui l'abandonneront, si par son commandement il ne m'emploie autre part. Auquel commandement je serai toujours obéissant... $»^{13}$.

Le premier vœu que Tirant prononce devant le roi de France, en posant la main sur le livre sacré de l'Évangile, est le suivant : "Je fais vœu à Dieu et à tous les saints du paradis et à mon seigneur le duc de Bretagne, capitaine général de cette troupe, héraut du très excellent et très chrétien roi de France, d'être, moi, aujourd'hui, le premier qui sortirai en cette terre et le dernier qui s'en retirerai » (chap. 113). Le vœu de Tirant est immédiatement réalisé, puisqu'il est le premier chevalier à aller à terre pour combattre les Maures et le dernier à en revenir. "Et tous les chevaliers accomplirent leurs vœux ", dit le texte. L'immédiateté de l'accomplissement du vœu crée l'effet de surprise, prépare le redoublement du vœu et la parodie.

Les chroniqueurs de Philippe le Bon citent avec précision les différents vœux des chevaliers: "Presque tout ce qu'il y avait là de princes et de grands seigneurs voua, sur l'oiseau, des prouesses immenses: tel de ne point boire de vin, tel autre de ne point s'asseoir à table ou de ne point se coucher ou de ne point revêtir de chemise un jour de la semaine, jusqu'à ce qu'il eût rencontré l'armée des infidèles, celui-ci de l'attaquer le premier; celui-là de renverser la bannière du sultan, un autre de ne pas revenir en Europe sans ramener un turc prisonnier... $»^{14}$. Mathieu d'Escouchy rapporte que le célèbre Pedro Vázquez de Saavedra - Piettre Vaast de Saibreda -, que les historiens s'accordent à 
reconnaitre comme un autre modèle de Tirant, fait vœu « aux dames et au faisan » que, "pour l'amour de Dieu », s'il lui arrive bataille, il abordera la bannière ou enseigne du Grand Turc, en telle manière qu'il l'abaissera...

Ces vœux qui mêlent les références religieuses et les références courtoises se retrouvent très exactement dans les chapitres 203 à 207 de Tirant lo Blanc. Mais dans le roman valencien, ce n'est pas sur l'oiseau symbolique des prouesses chevaleresques et évocateur de l'exotique pays de la Toison d'Or que les vœux sont prononcés, mais sur un esclave maure. Le redoublement des vœux à l'intérieur du roman fait signe vers un autre redoublement qui est l'épreuve de l'histoire bourguignonne et de l'histoire de l'Ordre de la Toison d'Or, par différenciation, par glissement parodique. De fait, c'est bien un glissement qui est à l'origine de ce second vœu. L'impératrice, désireuse de se moquer du puissant et conquérant Tirant, a en effet demandé à l'esclave qui doit porter Tirant d'une barque sur la rive de trébucher dans l'eau, afin de mouiller les pieds de Tirant qui sont revêtus de belles sandales brodées. «Il voulut lui mouiller le pied et il lui mouilla tout le corps... Tirant se rendit compte qu'on avait voulu se moquer de lui» (chap. 202). Tirant, complètement trempé, puissant mais tourné en dérision, prononce alors, en posant son pied sur la tête de l'esclave maure, le vœu suivant : «Je fais vœu à Dieu et à la demoiselle à qui j'appartiens de ne pas dormir dans un lit ni revêtir de chemise jusqu'à ce que j'ai fait mort ou prisonnier un roi ou un fils de roi » (chap. 203). Après lui, chacun de sa parentèle fait un vœu «par amour de lui », comme le constate Tirant. Les liens d'« amour » entre Tirant et ses parents s'inscrivent en contrepoint de la parodie du vœu chevaleresque de croisade. Est-ce un jugement porté ici par l'auteur du roman sur le politique Vœu du Faisan qui n'a abouti à aucune reconquête de Constantinople mais qui a renforcé les liens des vassaux envers leur suzerain?

41 Le vicomte de Branches, son « cousin germain ", au nom explicitement portugais ${ }^{15}$, parle après Tirant et dit : «Je fais vœu solennel à Dieu et à tous les saints de ne rentrer jamais dans ma propre terre sans avoir combattu quarante mille maures et les avoir vaincus... » (chap. 204). Le connétable Diafébus dit : « Je fais vœu à Dieu et à la noble dame dont je suis le captif de porter la barbe, de ne pas manger de viande ni m'asseoir à table, tant que je n'aurai pas pris la bannière du Grand Sultan au cours d'une bataille... » (chap. 205), ce qui rappelle très exactement le vœu de Pedro Vázquez de Saavedra. Hipolite, le très cher neveu de Tirant, dit: «Je fais vœu de ne manger ni pain ni sel, et d'être toujours agenouillé lorsque je mangerai, et de ne pas dormir dans un lit tant que je n'aurai pas tué trente maures de mes propres mains sans l'aide de personnes... » (chap. 206) ${ }^{16}$. Le Maure, sur qui ont été prononcés les vœux, est alors libéré par Tirant et richement doté de « diamants, rubis et perles ». Le faisan du banquet de Lille n'est-il pas lui-même "orné d'un très riche collier d'or, très richement garni de pierreries et de perles » (Mémoires d'Olivier de La Marche), cependant que l'Église captive quitte le banquet dans sa forteresse tirée par le géant " vêtu comme un sarrazin de Grenade » ?

Ce sont ces vœux de Tirant et de sa parentèle, qui trouvent leur réel accomplissement et non ceux du duc de Bourgogne et de ses chevaliers. Grâce à Tirant et ses compagnons, Constantinople échappe aux infidèles, l'empire chrétien d'Orient perdure cent ans, le temps de l'histoire est suspendu ! 


\section{Conclusion}

43 mariage avec Isabelle de Portugal, entouré de conseillers qui sont des hommes de droit et
de mémoire, acquiert la conviction en cette fin du $x^{\mathrm{e}}$ siècle qu'il est important de compléter le maniement des armes par celui de la parole chevaleresque : il récupère le vœu ancien et chevaleresque de la croisade pour en faire un serment de fidélité bien propre à assurer la cohésion de ses États. Cependant qu'un des derniers chevaliers errants, le Valencien Joanot Martorell, rompu à l'art des batailles et des querelles, donne à Tirant la gloire des mots et de la fiction narrative de la reconquête de l'empire chrétien d'Orient.

Le roman de Tirant souligne l'impuissance du grand duc d'Occident et des chevaliers de la Toison d'Or qui ne peuvent arrêter la progression des Ottomans vers l'Occident ni les empêcher de dominer la Méditerranée. Mais la parole chevaleresque n'a-t-elle pas été détournée par le grand duc d'Occident pour son désir et pour son plaisir ? Le souci des chroniqueurs des ducs ne consiste-t-il pas seulement à exalter la puissance de la Maison de Bourgogne? La seule gloire réelle et durable de la reconquête de l'empire chrétien d'Orient est donc attribuée au héros fictif de l'écriture chevaleresque et à son histoire. Le roman du chevalier valencien qui ne combat jamais en Orient vient se superposer, à son efficace mesure qui est celle du langage, sur les ambitions méditerranéennes de la Maison de Bourgogne.

Le Vœu du Faisan souligne la puissance occidentale du duc. Le roman de Tirant lo Blanc affirme que l'empire chrétien d'Orient n'a jamais été perdu, puisqu'il est sauvé pour cent ans par Tirant. Dans le présent toujours présent de sa lecture, le roman de Tirant prouve que la reconquête de l'empire chrétien d'Orient est un souvenir dont on se souvient au présent, une métaphore de l'absence. Car il ne convient plus depuis longtemps de reconquérir le tombeau vide du Christ à Jérusalem, comme l'a bien compris Philippe le Bon, ni même de s'attacher désormais à la possession chrétienne de Constantinople.

Tirant lo Blanc meurt sans être devenu empereur de Constantinople, alors que l'empereur lui a donné sa fille unique en mariage. Son corps et celui de l'héritière byzantine rejoignent la terre occidentale et arthurienne de l'origine du héros qui est aussi l'origine du récit ; ils sont, à la fin du roman, enclos dans un tombeau de Bretagne. En 1467, lorsque Philippe le Bon meurt, tous ses vassaux croisés se dispersent et Charles le Téméraire demande la couronne impériale en échange de son aide contre les infidèles. Le duché du grand duc d'Occident qui était l'interlocuteur favori de l'empereur byzantin, désormais mort, et en quelque sorte son égal, sera bientôt enclos dans le royaume de France. La dignité de souverain de l'Ordre de la Toison d'Or passera au chef de la Maison de Habsbourg et à Charles Quint. L'ordre est voué désormais à réaliser la fraternité de la noblesse des États de la Maison de Habsbourg. Le grand duc d'Occident n'a pas sauvé l'empire chrétien d'Orient, mais Charles Quint, son héritier, est empereur en Occident. Le vœu est bel et bien détourné pour le plus grand désir et le plus grand plaisir de la Maison de Bourgogne.

Dans la dédicace du Tirant, Joanot Martorell a défini en ces termes le projet du livre : «Il donnera vraiment à la chevalerie morale une lumière et lui représentera des exemples des bons usages, abolissant le tissu des vices et la cruauté des actes sanguinaires. » Un autre détournement s'effectue dont les faits ont établi la nécessité : si la quête 
chevaleresque de Jérusalem est, au début des aventures de Tirant, échangée en quête de Constantinople, il s'avère que Tirant meurt sans être empereur et que c'est dans un tombeau de Bretagne que se termine son histoire, là où elle a commencé. Il ne faut pas chercher de tombeau ni de fin en Orient. A partir de 1453, l'empire chrétien d'Orient est irrémédiablement perdu. Les vœux efficaces de Tirant et ses compagnons ne servent en fin de compte qu'à démontrer que la recherche de toute origine et de toute fin, de la lumière et du bien, se situe désormais dans l'Occident chrétien.

\section{NOTES}

1. Dans mon article «Tirant lo Blanc, le meilleur roman du monde : écriture et impression d'un roman de chevalerie en Catalogne après la disparition de l'empire chrétien d'Orient ", The Journal of Medieval and Renaissance Studies, XXI, 1991, p. 103-128, j'ai posé la question de l'auteur et de l'acte d'écriture du roman et je me suis intéressée à la succession des éditions.

2. Les lettres de bataille de Joanot Martorell ont été publiées par M. de Riquer dans l'introduction à son édition de Tirant lo Blanch, Barcelone, Ed. Selecta, Biblioteca Perenne, 1947. M. Vargas Llosa, Lletra de batalla per Tirant lo Blanc, Barcelone, Ed. 62, 2 éd., 1985.

3. Chronique de Jacques de Lalaing par Georges Chastellain, Paris, J.A.C. Buchon, 1825. Le Livre des faits de Jacques de Lalaing, longtemps attribué à Georges Chastellain et à Antoine de la Sale, est aujourd'hui considéré comme anonyme.

4. P. Champollion-Figeac, Documents inédits sur l'histoire de France, Paris, 1879, IV, p. 457-462.

5. Mathieu d'Escouchy, Chronique, Paris, éd. G. Du Fresne de Beaucourt, 1863, II, p. 160. Olivier de La Marche, Mémoires, éd. H. Beaune et J. d'Arbaumont, Paris, 1884, II, p. 382.

6. Depuis 1291 , avec la chute d'Acre, et 1303 , avec la chute de Gibelet, les dernières places franques de Terre sainte sont tombées. La croisade est la préoccupation majeure des papes, qui y voient volontiers une possibilité de réaliser la paix en Italie et en Europe, mais les différents projets n'aboutissent pas. Si, en 1328, un compromis avec le sultan permet aux pèlerins chrétiens de visiter le Saint Sépulcre, dès 1333 il s'avère que c'est un échec. Dès 1362-1366, les Turcs ottomans cherchent à s'emparer de Byzance. Le projet de croisade en Terre sainte se transforme en multiples combats contre les Turcs qui menacent l'Europe. Il s'agit désormais de sauver Constantinople, l'empire chrétien d'Orient et surtout les positions franques en Méditerranée. Tout au long $\mathrm{du} \mathrm{Xv}^{\mathrm{e}}$ siècle, les événements provoquent les appels successifs à la croisade par les papes. A partir de 1453, prise de Constantinople par Mohamed II, l'empire est détruit et Constantin XI Paléologue est mort. Mais lorsque le duc meurt en 1467, tous ses vassaux croisés se dispersent. Et l'auteur de Tirant lo Blanc meurt en 1468.

7. C. Marinesco, «Du nouveau sur Tirant lo Blanch ». Estudis Romànics, IV, 1953-1954, p. 137-204. Cet auteur analyse divers aspects du roman en les replaçant dans l'histoire contemporaine, et il propose comme modèle de Tirant, Jean Hunyade.

8. Jean de Wavrin possède d'importantes seigneuries en Artois et dans la châtellenie de Lille. En 1444, alors qu'il se trouve avec son oncle Walérand de Wavrin à Constantinople afin d'aider l'empereur, ce dernier pour les remercier offre à Walérand une relique qui lui permet d'organiser un pèlerinage dans sa seigneurie de Lillers.

9. Les citations sont données à partir du Ms. fr. 15 491, Bibliothèque nationale de France, inachevé, comportant seulement les chapitres de la chronique de Jean de Wavrin, relatifs aux 
années 1451-1471 qui nous importent ici, dans la mesure où elles correspondent à la fin des prouesses du chevalier blanc, à l'écriture de Tirant par Joanot Martorell et à la fin de la vie de Philippe le Bon $(\dagger 1467)$ et $d$ 'Isabel de Portugal ( $†$ 1471). Ce manuscrit provient de la bibliothèque des ducs de Bourgogne.

10. Les victoires de Jean Hunyade sont célébrées dans tout l'Occident et donnent lieu à des fêtes d'action de grâce à Valence et Barcelone. Le Dietari del capellà d'Alfons el Magnànim mentionne « lo comte Blanch », le Libre de solemnitats de Barcelona de 1456 mentionne « lo rey Blach » et le Manuel de novells ardits ou Dietari del Conseil de Barcelone, également de 1456, mentionne tout simplement « lo Blach ». L'écu porté par Jean Hunyade représente un corbeau et c'est pourquoi son fils prend le nom de "Corvin". Martorell, contemporain de Jean Hunyade, écrit que Tirant se fit faire « une bannière toute vermeille, et il y fit peindre un corbeau ", chap. 125.

11. M. de Riquer, Aproximació..., L'entremès del rei Artús, p. 150-156. Et surtout: A.G. Hauf, Artur a Constantinoble : entorn a un curiós episodi del Tirant lo Blanc, Alicante, 1994.

12. Il est remarquable que Joanot Martorell est très bien informé des cérémonies et des usages des cours les plus somptueuses d'Europe et en particulier de la cour de Bourgogne; décrivant un banquet à la cour de Constantinople, il rapporte : "Comme on était au milieu du dîner, Tirant donna aux rois d'armes, hérauts et poursuivants mille ducats. Et toutes les trompettes sonnèrent, et ils vinrent devant la table de l'empereur et ils criaient: Largesse, largesse!» (chap. 162). Olivier de la Marche écrit que, lors des fêtes des noces de Charles le Téméraire, «sur la fin du dîner se levèrent rois d'armes et hérauts... et vinrent crier devant la personne de monsieur le Duc : Largesse ! ", Collection universelle des mémoires particuliers relatifs à l'histoire de France, Mémoires d'Olivier de la Marche, II, Paris, éd. de J.A.C. Buchon, p. 569.

13. M. d'Escouchy, Chronique, chap. CIX, Paris, éd. G. Du Fresne de Beaucourt, II, 1863. Le récit par M. d'Escouchy de la fête du Faisan est presque identique à celui d'O. de La Marche et du Ms. Baluze 10319 .

14. O. de La Marche, Mémoires, IX, Londres, 1785, p. 19.

15. M. Sommé, Isabelle de Portugal duchesse de Bourgogne, une femme au pouvoir au $\mathrm{XV}^{e}$ siècle, Université de Lille III, 1995. L'auteur a bien montré l'importance des Portugais à la cour de Bourgogne et la manière dont leurs patronymes ont souvent été francisés. Rui de Pina, dans sa Crónica d'Alfonso V, chap. 31; Inéditos de história portuguesa, I, Lisbonne, 1790, p. 250, évoque le portuguais Alvaro Vaz de Almada qui servit le roi Henri V d'Angleterre et «pour sa hardiesse et ses mérites il fut fait comte d'Abranxes, et en Angleterre, pour sa vaillance, il fut reçu comme compagnon dans l'Ordre de la Jarretière ». Son fils, Joan de Almada, comte d'Abranxes, est dénommé dans les documents catalans "de Branches » M. de Riquer, La batalla a ultranza entre Joâo de Almada y Menaut de Beamont, Homenaje a Jaime Vicens i Vives, I, Barcelone, 1965, p. 579-590.

16. Tous ces vœux évoquent ceux du seigneur de Pons en Poitou, du comte d'Éstampes, de Jean de Luxembourg, de Jean de Bos ou du Bois, de Josse de Halwin et Gaules du Fossé, de Philippe Pot, de Hue de Longueval, d'Antoine de Ray, de Piettre Vaast de Saibreda, de Phelippe de Noyelle, d'Antoine de Loumay, de Jacques de Montmartin, Guillaume de Saux et Pierre de Haquembarc, de Jean de Chassa, de Loys du Chevalet, de Jean de Sailly, de Guillaume de Martigny, de Phelippe de Scoennehoves, de Jean Grignaut, d'Olivier de La Marche, Chronique de Mathieu d'Escouchy. 


\section{AUTEUR}

DOMINIQUE DE COURCELLES

CNRS, Paris 